Published in final edited form as:

Acupunct Med. 2020 August ; 38(4): 281-283. doi:10.1177/0964528420905841.

\title{
Acupuncture as an adjunctive therapy for stable angina
}

\author{
Holli A DeVon ${ }^{1}$, Judith M Schlaeger ${ }^{2}$, Joan E Briller ${ }^{3}$ \\ ${ }^{1}$ School of Nursing, University of California Los Angeles, Los Angeles, CA, USA \\ ${ }^{2}$ Department of Women, Children and Family Health Science, University of Illinois at Chicago, \\ Chicago, IL, USA \\ ${ }^{3}$ Department of Cardiology, University of Illinois at Chicago, Chicago, IL, USA
}

\section{Background}

There has been an impressive $32.7 \%$ decline in mortality from ischemic heart disease (IHD) since $1999 ;{ }^{1}$ however, the prevalence of stable angina has decreased in non-Hispanic Whites but not Blacks. ${ }^{2}$ In approximately $50 \%$ of cases, stable angina is the initial manifestation of IHD. Nearly a third of those afflicted report at least one symptom, and $23 \%$ report daily or weekly symptoms despite guideline-directed treatment. Angina in men is more frequently due to epicardial coronary obstruction. ${ }^{1}$ Humphries et al. ${ }^{3}$ found that $7.1 \%$ of males versus $23.3 \%$ of females in a large cohort $(n=32,856)$ of Canadians undergoing elective angiography for suspected angina symptoms had angiographically normal coronary arteries. A diagnosis of normal coronary vasculature or non-obstructive disease has been reported to be as much as five times more common in women. Many of these women are thought to have angina caused by microvascular coronary dysfunction, which may be attributed to hypertensive heart disease, hypertrophic cardiomyopathy, valvular heart disease, or multiple risk factors that result in arteriolar remodeling in the microvasculature. ${ }^{1}$ Women with nonobstructive disease have poorer outcomes than men. This is important because anti-anginal medications maybe less effective in the presence of microvascular coronary dysfunction. Hence, patients with symptomatic microvascular coronary dysfunction may benefit more from acupuncture therapy. In addition, it remains unclear if intensive medical therapy reduces major adverse cardiovascular events in women with non-obstructive disease. The ongoing Women's Ischemia (WARRIOR) trial may help answer this question.

\section{Physiologic mechanisms of acupuncture and their effects on the cardiovascular system}

Stable angina is associated with impaired functional status and reduced health-related quality of life. Acupuncture has demonstrated physiologic analgesic effects for individuals

\footnotetext{
Article reuse guidelines: sagepub.com/journals-permissions

Corresponding author: Holli A DeVon, School of Nursing, University of California Los Angeles, Factor Building, 700 Tiverton Avenue, Los Angeles, CA 90095, USA. hdevon@ sonnet.ucla.edu.

Declaration of conflicting interests

The authors declared no potential conflicts of interest with respect to the research, authorship, and/or publication of this article.
} 
with stable angina. Acupuncture regulates the autonomic nervous system and reduces sympathetic stimulation to the heart and vasculature by modulating the midbrain, releasing endorphins and dynorphins, with a resultant decrease in production of norepinephrine and epinephrine. ${ }^{4}$ These processes impact the cardiovascular system by reducing blood pressure, heart rate, and centrally mediated arrhythmias in the brainstem, as well as modulating the autonomic nervous system. One proposed mechanism of action for analgesic effects is the downregulation of M1 macrophages, interleukin (IL)-1 $\beta$, IL-6, IL-18, and tumor necrosis factor alpha (TNF-a). Many of these mechanisms of action may be similar to traditional pharmacologic treatment such as beta-blockers, which reduce blood pressure and myocardial oxygen demands.

Acupuncture use is on the rise in the United States. In 2012, Americans received 3,484,000 acupuncture treatments, with a significant increase documented from 2002 to $2007 .{ }^{5} \mathrm{In}$ 2012, 34\% of American adults used some form of complementary or alternative medicine (CAM). ${ }^{5}$ In 2007, $\$ 33.9$ billion dollars was spent on CAM. Most Americans use CAM to supplement (rather than replace) Western medical treatments and/or to treat symptoms of chronic disease or side effects from medications. Almost 8\% of those using CAM did not tell their physicians. Acupuncture has been recommended as a potential complementary or adjunctive treatment for hypertension, hyperlipidemia, and smoking cessation, but has not been proposed for stable angina in the United States. The 2012 joint American College of Cardiology Foundation (ACCF), American Heart Association (AHA), American College of Physicians (ACP), American Association for Thoracic Surgery (AATS), Preventive Cardiovascular Nurses Association (PCNA), Society for Cardiovascular Angiography and Interventions (SCAI) and Society of Thoracic Surgeons (STS) "Guideline for the Diagnosis and Management of Patients with Stable Ischemic Heart Disease" does not recommend acupuncture due to a paucity of studies, particularly a lack of double-blind randomized controlled trials (RCTs) ${ }^{6}$ There are strong justifications for studying acupuncture for angina including testing a potential complementary therapy for adults with inadequate pain and symptom control, as well as targeting a population of significant public health interest, namely three-million adults with stable angina whose symptoms are inadequately controlled with conventional therapy. In addition, the National Institutes of Health have recommended acupuncture as an alternative or complementary therapy for a number of conditions.

\section{Acupuncture for stable angina}

A recent systematic review of $25 \mathrm{RCTs}^{7}$ and a meta-analysis of eight RCTs ${ }^{8}$ of acupuncture for the treatment of stable angina showed relief of angina symptoms for acupuncturetreated participants compared to controls. However, there was no reduction in ischemia, as measured by electrocardiography, or reduction in nitroglycerin use. JAMA Internal Medicine just published the results of a rigorous four-arm clinical trial conducted by Zhao et al. ${ }^{9}$ The study, which analyzed data on 398 participants, was conducted at five clinical sites in China. While there was a reduction in angina episodes in all groups, there was a significant reduction in anginal frequency and severity as well as anxiety and depressive symptoms over the 16-week study in the group receiving acupuncture on the so-called "disease-affected meridian" (DAM). Participants in the DAM group also saw significant improvements in physical functioning. The primary and secondary outcomes were comprehensive and 
robustly measured. A limitation was that the study was conducted only in China. It is clear that the study should be replicated in an American population due to significant cultural and healthcare differences between East and West, major gaps in knowledge of acupuncture, a lack of publications in English, a paucity of high-quality RCTs, and the lack of standardized acupuncture protocols in these trials. There is no literature on the impact of acupuncture on the pathophysiologic mechanisms of stable angina and how specifically acupuncture modifies pain and symptoms. We are currently pilot testing a standardized acupuncture prescription (Figure 1), similar to the protocol of Zhao et al., ${ }^{9}$ in a diverse sample of Americans in our Acupuncture to Improve Symptoms for Angina (AIMS-A) study. ${ }^{10}$

Symptom management, usually addressed by risk factor modification and therapy with betablockers, calcium channel blockers, nitrates, or ranolazine, is critical for reducing disability and improving quality of life for individuals with angina. Nonetheless, nearly one-third of patients with stable angina have suboptimal management of their pain, or serious side effects from medications, at a lifetime cost of over $\$ 1$ million. This is unacceptable, considering we have the potential to improve symptoms of angina with acupuncture for women and men. Acupuncture is a low-risk therapy with virtually no side effects, and treatment is now covered by Medicare in the United States. Acupuncture has the potential to reduce the frequency of anginal episodes, the severity of pain, and other associated symptoms of stable angina by offering therapy that is complementary to optimal medical management. It is critical that we conduct large-scale pragmatic trials in the United States to determine the effectivness of acupuncture for persistent angina symptoms.

\section{Funding}

The authors disclosed receipt of the following financial support for the research, authorship, and/or publication of this article: This publication was made possible by Grant Number R21 NRO17705 from the National Institutes of Health, National Institute of Nursing Research (NINR). Its contents are solely the responsibility of the authors and do not necessarily represent the official views of the NINR. The final peer-reviewed manuscript is subject to the National Institutes of Health Public Access Policy.

\section{References}

1. Benjamin EJ, Muntner P and Bittencourt MSJC. Heart disease and stroke statistics-2019 update: a report from the American Heart Association. Circulation 2019; 139(10): e56-e528. [PubMed: 30700139]

2. Will JC, Yuan K and Ford E. National trends in the prevalence and medical history of angina: 1988 to 2012. Circ Cardiovasc Qual Outcomes 2014; 7(3): 407-413. [PubMed: 24847083]

3. Humphries KH, Pu A, Gao M, et al.Angina with "normal" coronary arteries: sex differences in outcomes. Am Heart J2008; 155(2): 375-381. [PubMed: 18215611]

4. Headrick JP, Pepe S and Peart JN. Non-analgesic effects of opioids: cardiovascular effects of opioids and their receptor systems. Curr Pharm Des 2012; 18(37): 6090-6100. [PubMed: 22747541]

5. Clarke TC, Black LI, Stussman BJ, et al.Trends in the use of complementary health approaches among adults: United States, 2002-2012. Natl Health Stat Report2015(79): 1-16.

6. Fihn SD, Gardin JM, Abrams J, et al.2012 ACCF/AHA/ACP/AATS/PCNA/SCAI/STS Guideline for the diagnosis and management of patients with stable ischemic heart disease: a report of the American College of Cardiology Foundation/American Heart Association Task Force on Practice Guidelines, and the American College of Physicians, American Association for Thoracic Surgery, Preventive Cardiovascular Nurses Association, Society for Cardiovascular Angiography and Interventions, and Society of Thoracic Surgeons. J Am Coll Cardiol2012; 60(24): e44-e164. [PubMed: 23182125] 
7. Yu C, Ji K, Cao H, et al.Effectiveness of acupuncture for angina pectoris: a systematic review of randomized controlled trials. BMC Complement Altern Med2015; 15: 90. [PubMed: 25888363]

8. Zhang Z, Chen M, Zhang L, et al.Meta-analysis of acupuncture therapy for the treatment of stable angina pectoris. Int J Clin Exp Med2015; 8(4): 5112-5120. [PubMed: 26131084]

9. Zhao L, Li D, Zheng H, et al.Acupuncture as adjunctive therapy for chronic stable angina: a randomized clinical trial. JAMA Intern Med2019; 179(10): 1388-1397. [PubMed: 31355870]

10. Schlaeger J, Cai HY, Steffen AD, et al.Acupuncture to improve symptoms for stable angina: protocol for a randomized controlled trial. JMIR Res Protoc2019; 8(7): e14705. [PubMed: 31359872] 


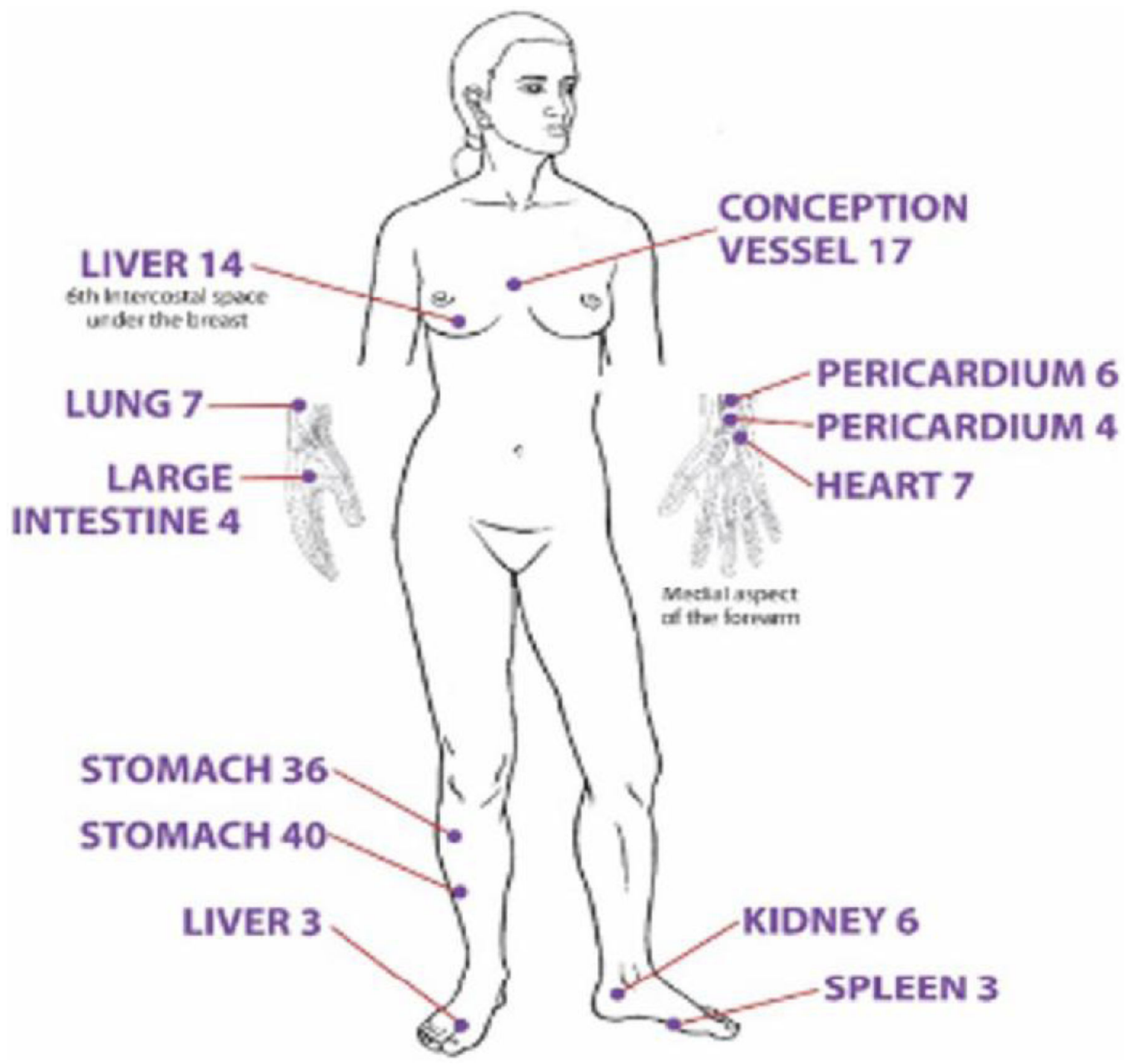

Figure 1.

Standardized acupuncture protocol for the treatment of stable angina. 